Final version published in Journal of Theoretical Biology 249 (1), 153-161 (2007)

\title{
Introduction of Trojan sex chromosomes to boost population growth
}

\author{
Samuel Cotton*, Claus Wedekind \\ Department of Ecology and Evolution, University of Lausanne, Biophore, CH - 1015 \\ Lausanne, Switzerland \\ * Corresponding author. Tel.: +41 (0)21 69242 03; fax: +41 (0)21 6924165 . \\ Email addresses: samuel.cotton@unil.ch (S. Cotton), claus.wedekind@unil.ch \\ (C. Wedekind).
}

\begin{abstract}
Conservation programs that deal with small or declining population often aim at a rapid increase of population size to above critical levels in order to avoid the negative effects of demographic stochasticity and of genetic problems like inbreeding depression, fixation of deleterious alleles, or a general loss of genetic variability and hence of evolutionary potential. In some situations, population growth is determined by the number of females available for reproduction, and manipulation of family sex ratios towards more daughters has beneficial effects. If sex determination is genetic and environmental, as is the case in many amphibia, reptiles, and fish, Trojan sex chromosomes could be introduced into populations in order to change sex ratios towards more females. We analyse the possible consequences for the introduction of $X X$-males ( $X X$ individuals that have been changed to phenotypic males in a XY / XX sex determination system) and ZW males, WW males, or WW females (in a ZZ/ZW sex determination system). We find that the introduction of WW individuals can be most effective for an increase of population growth, especially if the induced sex change has little or no effect on viability.
\end{abstract}

Keywords: effective population size; female heterogamety; sex ratio; small population; Trojan sex chromosome; amphibia; fish; supportive breeding; captive breeding 


\section{INTRODUCTION}

Populations that decrease in size and especially those that stay at low numbers over several generations have a reduced long-term survival expectancy due to stochasticity effects and several genetic problems (Lande, 1998; Courchamp et al., 1999; Stephens and Sutherland, 1999). The latter include an increase in inbreeding and any associated inbreeding depression, an increased risk of fixation of deleterious mutations due to random drift, and a general loss of genetic variability and therefore a reduced potential for adaptation to changing environments (Frankham et al., 2002). The rate with which a population loses genetic variability depends on $N_{e}$, the genetically effective population size. $N_{e}$ is the size of an ideal population that loses genetic variability at the same rate as the real population (Falconer and Mackay, 1996). With lower $N_{e}$, more genetic variability is lost from generation to generation.

In some cases, the number of offspring per female is a limiting factor with regard to population growth. In such cases, it may be possible to amplify population growth (and finally achieve an increase of $N_{e}$ ) by manipulating family sex ratios and producing a higher ratio of the reproductively limiting sex (Tella, 2001; Clout et al., 2002; Wedekind, 2002; Robertson et al., 2006; Lenz et al., 2007). Such manipulation is often possible, either through noninvasive techniques like changing sex-determining ecological or social factors (Roberge et al., 2006), or through more invasive techniques such as hormone treatment of embryos or sperm sexing prior to using assisted reproductive technologies (Wildt and Wemmer, 1999; Pukazhenthi et al., 2006). The aim is to lower the sex ratio, defined here as the proportion of males, and hence to increase the production of females if they are the limiting factor for population growth.

Male-biased sex ratios have recently been modelled as a strategy for controlling the population sizes of non-native alien species. Gutierrez and Teem (2006; see also Cotton \& Wedekind, submitted) proposed the deployment of females containing "Trojan" sex chromosomes as a method for eliminating problem populations with male heterogamety (i.e. $X Y=$ males, $X X=$ females). Trojan sex chromosome carriers are individuals who are phenotypically sex reversed from their genotype (i.e. XY or YY females), usually as a result of treatment with sex hormones during early development. When these individuals are released into a wild population, they mate with wild-type $(\mathrm{XY})$ males and cause a male-biased sex ratio in subsequent generations owing to the disproportionate influx of one sex chromosome. After repeated introduction of Trojan sex chromosome carriers, true (XX) females become increasingly rare and the population may become extinct.

Here, we show that the logic of Trojan sex chromosomes can be extended to cause benefit to small populations with female heterogamety (i.e. $\mathrm{ZZ}=$ males, $\mathrm{ZW}=$ females); no such advantages were found for male heterogametic systems. We demonstrate how the release of Trojan sex chromosome carriers (e.g. WW females or WW males) can cause a femalebiased sex ratio over many generations, and hence a disproportionate increase in both the absolute and effective population size, when compared to augmentation of the population with the same number of normal females. We show that these advantages persist even when the released Trojan sex chromosomes carriers have slightly lower viability than wild-type individuals. We believe that our proposed use of Trojan sex chromosomes as 
a positive force in conservation biology may be particularly applicable to the amphibia, as they are undergoing a major worldwide decline in abundance, and represent an important conservation priority (Houlahan et al., 2000; Stuart et al., 2004; Beebee and Griffiths, 2005). Female heterogamety is widespread in the amphibia (Hillis and Green, 1990), and the sex of many species in this taxon can be manipulated by changing environmental conditions during early development (see below).

The sex-reversed individuals required to deploy Trojan sex chromosomes can be produced by numerous means. Application of sex hormones (estrogens to feminize and androgens to maculinize) is used in many fish species displaying both male and female heterogamety (reviews in Pandian and Sheela, 1995; Devlin and Nagahama, 2002). In amphibians displaying female heterogamety, grafting of male gonadal tissue onto female embryos produces masculinised females in a number of species (e.g. axolotls, the newt Pleurodeles waltl, Xenopus laevis; reviewed in Wallace et al., 1999). Temperature can also be used to induce sex change; ZW P. waltl larvae incubated at $32^{\circ} \mathrm{C}$ develop as functional ZW males (Chardard and Dournon, 1999). More widely, varying the levels of aromatase, the enzyme that converts androgens to estrogens, and hence effects masculinization, is frequently been used as a method for manipulating sex in many taxa (e.g. fish, Devlin and Nagahama, 2002; birds, Elbrecht and Smith, 1992; reptiles, Pieau et al., 1999), including amphibians (Chardard and Dournon, 1999). The administration of aromatase inhibitors during early development therefore represents an easy and effective way of producing Trojan sex chromosome carriers.

\section{METHODS}

\section{Trojan sex chromosomes in a ZZ/ZW system}

We consider the use of three types of Trojan sex chromosome carrier: ZW males, WW females and WW males. ZW males can be produced by the phenotypic sex reversal of normal ZW females. WW females can be produced as one quarter of the progeny of $\mathrm{ZW}$ female $\times \mathrm{ZW}$ male crosses. These $\mathrm{WW}$ females can also be masculinised to produce WW males. These processes are summarised in Figure 1.

The model aims to evaluate changes in $N_{e}$ of a small population displaying female heterogamety. For simplicity, we assume that the population is characterised by discrete generations, semelparity and an initial, natural, sex ratio of 1:1. Females mate only once, and each contributes $n$ offspring to the subsequent generation; population growth is limited by production from females. Males can mate with more than one female if the sex ratio becomes female biased. Mating among males and females is random with respect to genotype. The population initially has $\mathrm{N}_{\mathrm{MzZ}} \mathrm{ZZ}$ males and $\mathrm{N}_{\mathrm{FZW}} \mathrm{ZW}$ females (actual population size, $N_{a}=N_{M}+N_{F}$ ), with

$$
N_{e}=\frac{4\left(\mathrm{~N}_{\mathrm{M} \mathrm{ZZ}} \mathrm{N}_{\mathrm{FZW}}\right)}{\left(\mathrm{N}_{\mathrm{M} \mathrm{ZZ}}+\mathrm{N}_{\mathrm{F} \mathrm{ZW}}\right)}
$$

(Falconer and Mackay, 1996). To model the effect on population growth of Trojan sex chromosomes, we introduce carriers into the population in generation $t=1$. We examine three types of individuals carrying Trojan sex 
chromosomes: (i) ZW males (frequency: $\mathrm{N}_{\mathrm{MZW}}$ ), (ii) WW males $\left(\mathrm{N}_{\mathrm{MWw}}\right)$ and (iii) WW females $\left(\mathrm{N}_{\mathrm{FWW}}\right)$. The population growth associated with each of these additions is compared against population growth attributable to the augmentation of the population with the same number of either wild-type $\mathrm{ZZ}$ males $\left(\mathrm{N}_{\mathrm{MzZ}}\right.$ ) or $\mathrm{ZW}$ females $\left(\mathrm{N}_{\mathrm{FZW}}\right)$. If individuals with the Trojan $\mathrm{W}$ chromosome are females, then they produce $n$ offspring, whose genotypes are distributed as in Figure 2. If they are males, then the expected frequencies of matings between each genotype are frequency-dependent, and given in Table 1 . Each of these matings produces $n$ offspring whose genotypes are distributed in the proportions given in Figure 2.

The recurrence equations for the frequencies of each genotype produced in generation $t+1$ are:

$$
\begin{aligned}
& \mathrm{N}_{\mathrm{MZZ}(\mathrm{t}+1)}=\left(0.5 \mathrm{na}_{(\mathrm{t})}\right)+\left(0.25 \mathrm{nc}_{(\mathrm{t})}\right), \\
& \mathrm{N}_{\mathrm{FZW}_{(\mathrm{t}+1)}}=\left(0.5 \mathrm{na}_{(\mathrm{t})}\right)+\left(\mathrm{nb}_{(\mathrm{t})}\right)+\left(0.5 \mathrm{nc}_{(\mathrm{t})}\right)+\left(0.5 \mathrm{nd}_{(\mathrm{t})}\right)+\left(0.5 \mathrm{ne}_{(\mathrm{t})}\right), \\
& \mathrm{N}_{\mathrm{FWW}_{(\mathrm{t}+1)}}=\left(0.25 \mathrm{nc}_{(\mathrm{t})}\right)+\left(0.5 \mathrm{nd}_{(\mathrm{t})}\right)+\left(0.5 \mathrm{ne}_{(\mathrm{t})}\right)+\left(\mathrm{nf}_{(\mathrm{t})}\right),
\end{aligned}
$$

where $a-f$ are expected mating frequencies between each genotype in generation $t$ (Table 1). $N_{e}$ after $t$ generations is given by the harmonic mean of $N_{e}$ in each previous generation(s) (Falconer and Mackay, 1996),

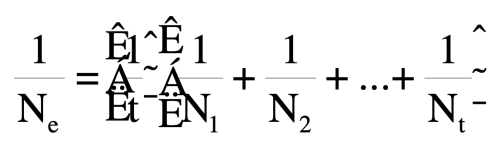

We simulated two introduction scenarios. First, a single release of Trojan sex chromosome carriers in generation $t=1$, and second, a release during each generation for five generations ( $t=1$ to 5$)$.

\section{Trojan sex chromosomes in a $\mathrm{XY} / \mathrm{XX}$ system}

Here we consider the use of $X X$ males that can be produced by the phenotypic sex reversal of normal XX females. The model aims to evaluate changes in $N_{e}$ of a small population displaying male heterogamety. We again work with populations that are characterised by discrete generations, semelparity and an initial, natural, sex ratio of 1:1. Females mate only once, and each contributes $n$ offspring to the subsequent generation (in the proportions specified in Figure 2); population growth is limited by production from females. Males can mate with more than one female if the sex ratio becomes female biased. Mating among males and females is random with respect to genotype. $N_{e}$ is corrected for unequal sex ratios (analogous to equation (1)), and over time as above (equation (2)), and we introduce carriers into the population in generation $t=1$ or during the first five generations.

The recurrence equations for the frequencies of each genotype produced in generation $t+1$ are:

$$
\begin{aligned}
& \mathrm{N}_{\mathrm{MXY}(\mathrm{t}+1)}=0.5 \mathrm{ng}_{(\mathrm{t})^{\prime}} \\
& \mathrm{N}_{\mathrm{FXX}_{(\mathrm{t}+1)}}=\left(0.5 \mathrm{ng}_{(\mathrm{t})}\right)+\left(\mathrm{nh}_{(\mathrm{t})}\right),
\end{aligned}
$$


where $g$ and $h$ are the expected mating frequencies between each genotype in generation $t$ (Table 1 ).

\section{The influence of Trojan carrier viability on population growth}

Sex-reversed individuals or those with an unnatural complement of sex chromosomes may suffer reduced viability relative to wild-type individuals. We explored the consequences on $N_{e}$ of introduced individuals having lower reproductive success. We assigned an independent fitness coefficient to Trojan carriers of each sex ( $p$ for males and $q$ for females), which scales the number of progeny derived from a mating. For example, a mating between ZW males and ZW females would produce $p n$ offspring, between WW females and ZZ males $q n$ offspring, and between WW females and WW males $p q n$ offspring. We then re-ran the simulations over varying values of $p$ and $q$ and assessed changes in $N_{e}$.

\section{RESULTS}

\section{Fitness of Trojan individuals $=$ fitness of wild-type individuals}

We simulated the addition of a small number of individuals (5\% of population) into a small population in generation $t=1$ (Figure $3 a, b)$. We compared changes in $N_{e}$ and actual population size $\left(N_{a}\right)$ after augmenting the population with individuals harbouring Trojan sex chromosomes (ZW males, WW females and WW males) vs. comparable additions of wild-types (ZZ males and ZW females). Assuming that the viability of Trojan individuals was equivalent to the wild-type (see below), the strategy of releasing WW individuals yielded a greater increase in $N_{a}$ and $N_{e}$ than introducing normal ZW females (or ZZ males, the least optimal strategy). Liberation of WW females into the population led to the greatest improvement in $N_{a}$ and $N_{e}$. Releasing ZW males increases $N_{e}$ but this strategy was always less successful than the simple release of normal ZW females (Figure 3). When groups of individuals were released into the population over five successive generations ( $t=1$ to 5 ; Figure $3 c, d)$, we found that WW individuals elevated $N_{a}$ and $N_{e}$ by the highest amount. Again, ZW males increased $N_{e}$, but not by as much as simply augmenting the population with normal ZW females.

In systems with male heterogamety, we found that the benefits of introducing Trojan sex chromosome carriers (XX males) into a small population were not as great as that of simple augmentation with normal $\mathrm{XX}$ females (Figure 4), although with repeated introduction over 5 generations $N_{a}$ was higher when Trojan carriers were added (Figure 4c). So addition of Trojan sex chromosome carriers is unlikely to be an optimal management strategy for small populations in male heterogametic systems.

The advantages of releasing Trojan sex chromosome carriers into populations with female heterogamety persisted irrespective of their initial introduction frequency (simulated from 1-20\% of population; Figure 5). However, the increase in $N_{e}$ (from an initial $N_{e}=100$ ) after release of Trojan sex chromosome carriers became more marked as the number of introduced individuals increased (Figure 5). Moreover, the release of WW females seemed to be slightly more effective when introduced during a single release episode, while the release of WW males was more effective when the 
introduction was repeated over five generations, especially so at high frequency (Figure 5).

The advantageous increases in $N_{e}$ and $N_{a}$ were attributable to changes in sex ratios after introduction of Trojan individuals (Figure 6). The population became female biased in the generations subsequent to both single (Figure 6a) and multiple (Figure 6b) introductions. The superiority of WW release strategies arose because of the greater persistence and magnitude of female sex ratio biases in those populations (Figure 6a,b).

\section{If fitness of Trojan individuals < fitness of wild-type individuals}

We simulated the effect on $N_{e}$ of a reduction of fitness (number of progeny) of individuals carrying Trojan sex chromosomes. Independent fitness coefficients were given to each sex. The consequences of reduced Trojan fitness were similar for both single and multiple releases (Figure 7). The success of the release of $\mathrm{ZW}$ males (although always inferior to the release of ZW females) was affected by the relative fitnesses of both the introduced Trojan males and the resultant Trojan females (Figures 7a,d). In contrast, effect on $N_{e}$ of WW female introduction was immune to reduced male viability (since no Trojan males are produced; Figures $7 \mathrm{~b}, \mathrm{e}$ ). However, the advantage of the WW female introduction method diminished as Trojan female fitness declined relative to wild-type individuals (Figures 7b,e); in our simulations, WW female fitness could reduce to one half before it became inferior to the simple release of ZW females. Population growth after release of WW males was severely tempered by reductions in both male and female Trojan fitness (Figures 7c,f). A decrease of $\sim 25 \%$ in male/female Trojan fitness abolished the advantage of WW male introduction over ZW female introduction (Figures 7c,f).

\section{DISCUSSION}

Small populations may often benefit from transient female-biased sex ratios, as this elevates population productivity and increases $N_{e}$ over the long-term (Tella, 2001; Wedekind, 2002). Here, we describe a novel method for effecting such sex ratio biases. The recent proposal, that individuals harbouring Trojan sex chromosomes can be used in conservation biology to control problem populations (Gutierrez and Teem, 2006; Cotton and Wedekind, submitted), is extended to show that such individuals can cause benefit to small populations in species with female heterogamety. Our simulations suggest that the strategy of augmenting a small population with WW-containing individuals is a productive method of increasing $N_{e}$. Trojan sex chromosomes may therefore be used as a positive force in conservation biology in such species. The introduction of Trojan sex chromosome carriers (XX males) is not the optimal strategy for elevating $N_{e}$ in populations with male heterogamety, although with repeated introductions over a number of generations such a strategy may lead to a marginally higher census size.

Female-biased sex ratios have previously been shown to elevate the effective size of populations whose growth is limited by female productivity (Tella, 2001; Clout et al., 2002; Wedekind, 2002; Robertson et al., 2006; Lenz et al., 2007). The Trojan sex chromosomes modelled in female heterogametic populations in this paper cause a disproportionate number of their carrier's offspring (and grand-offspring) to become female (Figure 2), and hence 
increase the productivity of the populations. The strategy of releasing Trojan sex chromosomes is superior to that of releasing wild-type $\mathrm{ZW}$ females because it causes a greater persistence and magnitude of female sex ratio biases. Our simulations show that the efficacy of the method increases as higher numbers of Trojan carriers are released into the population. However, even the release of small numbers can cause appreciable increases in $N_{\ell}$. We also note that while we have envisaged sex as being determined via distinct (e.g. W and Z) chromosomes, similar dynamics will be observed in systems where sex is primarily dictated by one or several major genes.

Our proposed use of Trojan sex chromosomes to increase $N_{e}$ may be particularly relevant for amphibian conservation efforts. Amphibians are facing extensive global declines (Houlahan et al., 2000; Stuart et al., 2004; Beebee and Griffiths, 2005) and many species exhibit the required female heterogamety (Hillis and Green, 1990) and are susceptible to phenotypic sex reversal (Wallace et al., 1999; Chadard and Dournon, 1999). The method may also be practical in other sexually plastic taxa with female heterogamety, and potentially even in birds (Elbrecht and Smith, 1992; Takagi et al., 2007).

One of the problems associated with the use of Trojan sex chromosomes is the potential for Trojan carriers to have lower fitness than wild-types, as a result of sex-reversal treatment and/or their unnatural complement of sex chromosomes (Cotton and Wedekind, submitted). We therefore incorporated such possible effects onto our models. As expected, we found that the success of the Trojan sex chromosome method was tempered when carriers suffered a fitness cost. Nonetheless, the release of Trojan sex chromosome $(\mathrm{WW})$ carriers still elevated $N_{e}$ beyond that observed with $\mathrm{ZW}$ female introduction provided that the reduction in fitness was modest $(\leq 25 \%$ in our simulations). The success of WW female introduction was contingent only on variation in Trojan female fitness $(q)$, being unaffected by reduced male fitness $(p)$; the release of WW females results only in female progeny. In addition, the release of WW females was less sensitive to reductions in Trojan fitness, being the optimal release strategy so long as the reduction of fitness of WW females was $<0.5$. Thus our simulations suggest that releasing WW females is the optimal and most robust strategy for increasing $N_{e}$. It also has the practical advantage that only one round of sex reversal is required during the production of Trojan sex chromosome carriers.

The magnitude of any reduced viability for Trojan sex chromosome carriers in the wild is unknown. However, a number of studies have reported that sex-reversed and $\mathrm{W}$ chromosome disomic individuals are viable in amphibians (reviewed in Wallace et al., 1999; Chadard and Dournon, 1999). This may be because most amphibia lack morphologically distinct sex chromosomes (Hayes, 1998), and hence the $W$ chromosome may still retain sufficient genes to enable full viability when inherited as a homozygous pair. This increases the efficacy and utility of Trojan sex chromosomes in this taxon.

As with Gutierrez and Teem's (2006) original model of Trojan sex chromosomes, our theory has yet to receive experimental verification. The requisite characteristics (environmentally-inducible sex change) exist in a number of species commonly used as laboratory models, so dynamics of Trojan sex chromosomes can be readily verified under controlled conditions, prior to their deployment in species with struggling real-world populations. Future empirical work is therefore required to test the assumptions of Trojan 
sex chromosome theory (Gutierrez and Teem, 2006; this paper), and its feasibility as a population growth regulator.

\section{Acknowledgements}

Financial support was provided by the Swiss National Science Foundation.

\section{References}

Beebee, T.J.C., Griffiths, R.A., 2005. The amphibian decline crisis: a watershed for conservation biology? Biol. Conserv. (125), 271-285.

Chardard, D., Dournon, C., 1999. Sex reversal by aromatase inhibitor treatment in the Newt Pleurodeles waltl. J. Exp. Zool. (283), 43-50.

Clout, M.N., Elliott, G.P., Robertson, B.C., 2002. Effects of supplementary feeding on the offspring sex ratio of kakapo: a dilemma for the conservation of a polygynous parrot. Biol. Conserv. (107), 13-18.

Cotton, S., Wedekind, C., submitted. Control of introduced species using Trojan sex genes. Trends Ecol. Evol.

Courchamp, F., Clutton-Brock, T., Grenfell, B., 1999. Inverse density dependence and the Allee effect. Trends Ecol. Evol. (14), 405-410.

Devlin, R.H., Nagahama, Y., 2002. Sex determination and sex differention in fish: and overview of genetic, physiological, and environmental influences. Aquaculture. (208), 191364.

Elbrecht, A., Smith, R.G., 1992. Aromatase enzyme activity and sex determination in chickens. Science (255), 467-470.

Falconer, D.S., Mackay, T.F.C., 1996. Introduction to quantitative genetics ( $4^{\text {th }}$ Ed.) Pearson, UK.

Frankham, R., Ballou, J.D., Briscoe, D.A., 2002. Introduction to conservation genetics. Cambridge: Cambridge University Press.

Gutierrez, J.B., Teem, J.L., 2006. A model describing the effect of sex-reversed YY fish in an established wild population: the use of a Trojan $Y$ chromosome to cause extinction of an introduced exotic species. J. theor. Biol. (241), 333-341.

Hayes, T.B., 1998. Sex determination and primary sex differentiation in amphibians: genetic and developmental mechanisms. J. Exp. Zool. (281), 373-399.

Hillis, D.M., Green, D.M., 1990. Evolutionary changes of heterogametic sex in the phylogenetic history of amphibians. J. Evol. Biol. (3), 49-64.

Houlahan, J.E., Findlay, C.S., Schmidt, B.R., Meyer, A.H., Kuzmin, S.L., 2000. Quantitative evidence for global amphibian population declines. Nature (404), 752-755.

Lande, R., 1998. Anthropogenic, ecological and genetic factors in extinction. In Conservation in a changing world: 29-51. G.M. Mace, A. Balmford, and J.R. Ginsberg, (Eds.). Cambridge: Cambridge University Press.

Lenz, T.L., Jacob, A., Wedekind, C., 2007. Manipulating sex ratio to increase population growth: the example of the Lesser Kestrel. Anim. Conserv. (10), 236-244.

Pandian, T.J., Sheela, S.G., 1995. Hormonal induction of sex reversl in fish. Aquaculture (138), $1-22$. 
Pieau, C., Dorizzi, M., Richard-Mercier, N., 1999. Temperature-dependent sex determination and gondadal differentiation in reptiles. CMLS, Cell. Mol. Life. Sci. (55), 887-900.

Pukazhenthi, B., Comizzoli, P., Travis, A.J., Wildt, D.E., 2006. Applications of emerging technologies to the study and conservation of threatened and endangered species.

Reproduction Fertility and Development (18), 77-90.

Roberge, C., Einum, S., Guderley, H., Bernatchez, L., 2006. Rapid parallel evolutionary changes of gene transcription profiles in farmed Atlantic salmon. Mol. Ecol. (15), 9-20.

Robertson, B.C., Elliott, G.P., Eason, D.K., Clout, M.N., Gemell, N.J., 2006. Sex allocation theory aids species conservation. Biology Letters (2), 229-231.

Stephens, P.A., Sutherland, W.J., 1999. Consequences of the Allee effect for behaviour, ecology and conservation. Trends Ecol. Evol. (14), 401-405.

Stuart, S.N., Chanson, J.S., Cox, N.A., Young, B.E., Rodrigues, A.S.L., Fischman, D.L., Waller, R.W., 2004. Status and trends of amphibian declines and extinctions worldwide. Science (306), 1783-1786.

Takagi, S., Tsukada, A., Saito, N., Shimada, K., 2007. Fertilizing ability of chicken sperm bearing the W chromosome. Poult. Sci. (86), 731-738.

Tella, J.L., 2001. Sex-ratio theory in conservation biology. Trends Ecol. Evol. (16), 76-77.

Wallace, H., Badawy, G.M.I., Wallace, B.M.N., 1999. Amphibian sex determination and sex reversal. CMLS, Cell. Mol. Life Sci. (55), 901-909.

Wedekind, C., 2002. Manipulating sex ratios for conservation: short-term risks and long-term benefits. Anim. Conserv. (5), 13-20.

Wildt, D.E., Wemmer, C., 1999. Sex and wildlife: the role of reproductive science in conservation. Biodiversity and Conservation (8), 965-976. 
Table 1. Mating frequencies of the different genotypes. Mating frequencies $a$ $h$ depict those as in Figure 1.

\begin{tabular}{|c|c|c|}
\hline Male genotype & Female genotype & Mating Frequency \\
\hline $\mathrm{ZZ}$ & $\mathrm{ZW}$ & $\mathrm{a}=\stackrel{\hat{\mathrm{E}}}{\stackrel{\hat{\mathrm{E}}}{\mathrm{N}_{\mathrm{M} \mathrm{ZZ}}}+\mathrm{N}_{\mathrm{M} \mathrm{ZW}}+\mathrm{N}_{\mathrm{MWw}}{ }^{-}} \stackrel{\hat{\sim}}{\sim \mathrm{N}_{\mathrm{FZW}}}$ \\
\hline $\mathrm{ZZ}$ & WW & $\mathrm{b}=\underset{\underset{\mathrm{E}^{\mathrm{E}}}{\hat{\mathrm{E}}}}{\mathrm{N}_{\mathrm{M} \mathrm{ZZ}}}+\mathrm{N}_{\mathrm{M} \mathrm{ZZ}}+\mathrm{N}_{\mathrm{MZW}}+\mathrm{N}_{\mathrm{MWw}}{ }^{-} \mathrm{N}_{\mathrm{FWW}}$ \\
\hline ZW & ZW & $\mathrm{c}=\underset{\underset{\mathrm{E}}{\hat{\mathrm{E}}}}{\hat{\mathrm{E}}} \mathrm{N}_{\mathrm{M} \mathrm{ZZ}}+\mathrm{N}_{\mathrm{MZW}}+\mathrm{N}_{\mathrm{MWw}}{ }^{-} \mathrm{N}_{\mathrm{FZW}}$ \\
\hline ZW & WW & 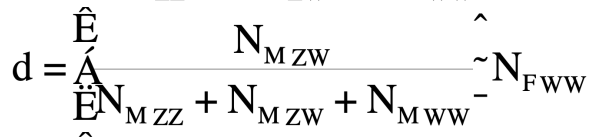 \\
\hline WW & ZW & 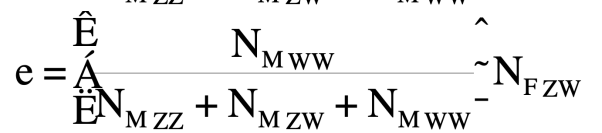 \\
\hline WW & WW & 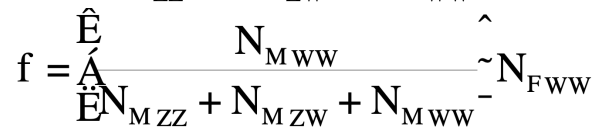 \\
\hline $\mathrm{XY}$ & $\mathrm{XX}$ & 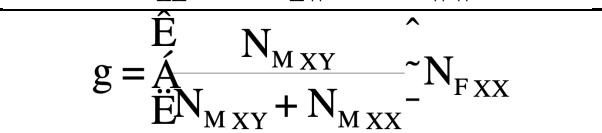 \\
\hline$X X$ & $X X$ & $\mathrm{~h}=\underset{\ddot{\mathrm{E}}^{\mathrm{E}}}{\hat{\mathrm{E}}} \mathrm{N}_{\mathrm{MXX}} \stackrel{\hat{\sim}}{\sim \mathrm{N}_{\mathrm{MXX}}} \mathrm{N}_{\mathrm{FXX}}$ \\
\hline
\end{tabular}


Figure 1. A schematic of production of Trojan individuals. Individuals in grey boxes are carriers of Trojan sex chromosomes.

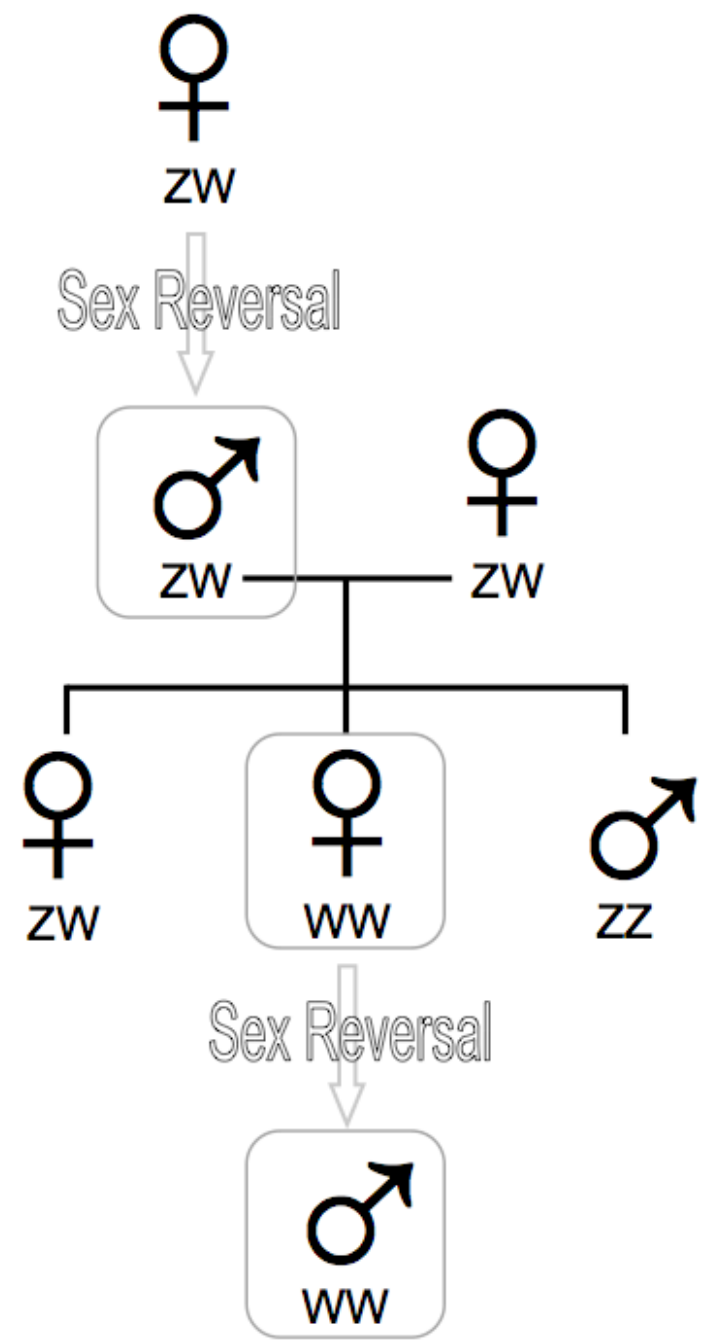


Figure 2. Pedigrees resulting from the mating of different genotype combinations: ( $\boldsymbol{a}$ ) wild-type ZZ males and ZW females, (b) wild-type ZZ males and Trojan WW females, (c) Trojan ZW males and wild-type ZW females, $(\boldsymbol{d})$ Trojan ZW males and Trojan WW females, $(\boldsymbol{e})$ Trojan WW males and wild-type females, $(f)$ Trojan WW males and Trojan WW females, $(g)$ wild-type XY males and XX females, and $(\boldsymbol{h})$ Trojan $X X$ males and wild-type $X X$ females. The numbers associated with each mating type depict the proportion of each clutch of each sex/genotype contribution. Individuals in grey boxes are carriers of Trojan sex chromosomes.

(a)

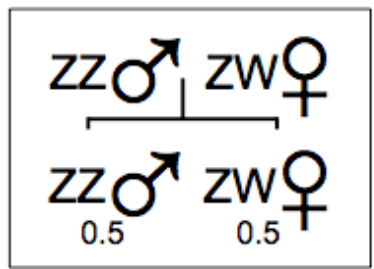

(c)

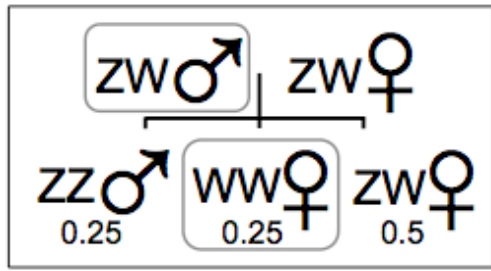

(e)

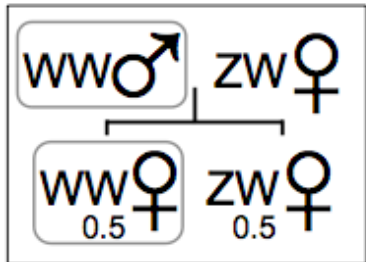

(b)

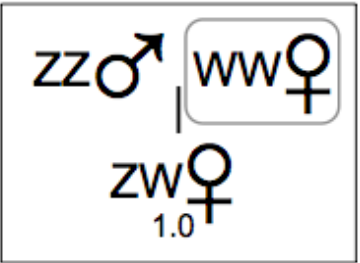

(d)

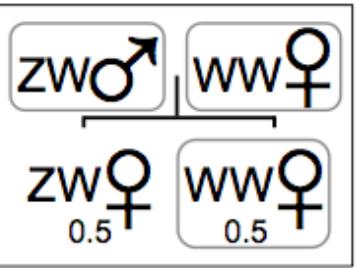

(f)

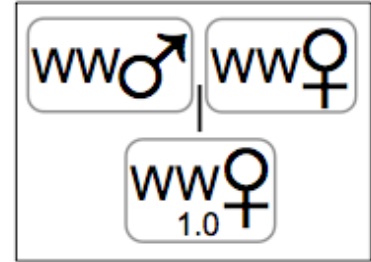

(g)

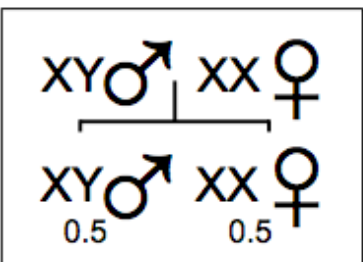

(h)

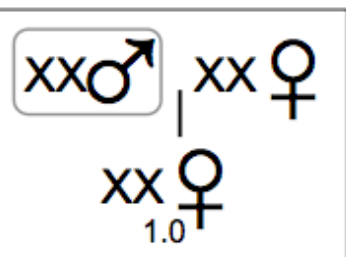


Figure 3. The effect of introducing individuals containing Trojan sex chromosomes $(N=5)$ into a small wild population $\left(\mathrm{N}_{\mathrm{MzZ}}=50, \mathrm{~N}_{\mathrm{FZW}}=50\right)$ on $N_{a}(\boldsymbol{a}, \boldsymbol{c})$ and $N_{e}(\boldsymbol{b}, \boldsymbol{d})$ and. Trojan individuals were introduced during $(\boldsymbol{a}, \boldsymbol{b})$ a single episode in generation $t=1$, and $(c, d)$ for five generations $(t=1$ to 5 ). Female productivity was set at $n=2$ offspring. Changes in population size are depicted after introduction of ZW males (crosses, black line), WW females (circles, black line) and WW males (squares, black line). For comparison, growth curves resulting from ZW female (circles, grey line) and ZZ male (squares, grey lines) introduction (extra $\mathrm{N}_{\mathrm{MZZ}}$ and $\mathrm{N}_{\mathrm{FZW}}=5$ ) are also depicted.

(a)

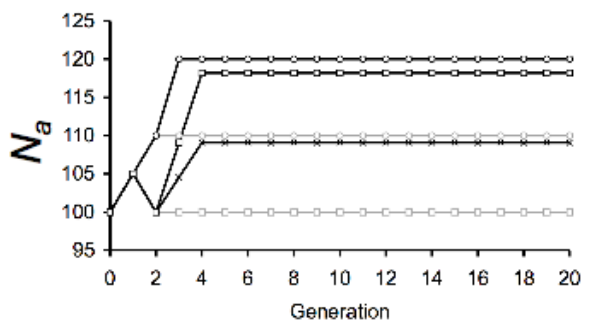

(c)

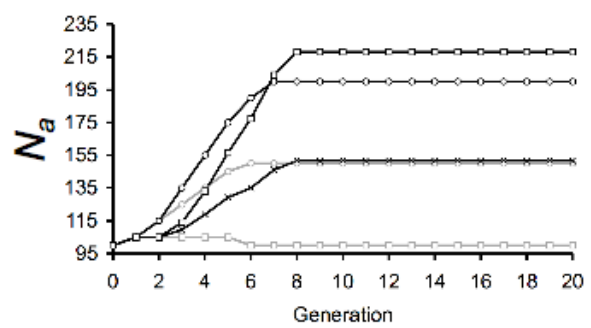

(b)

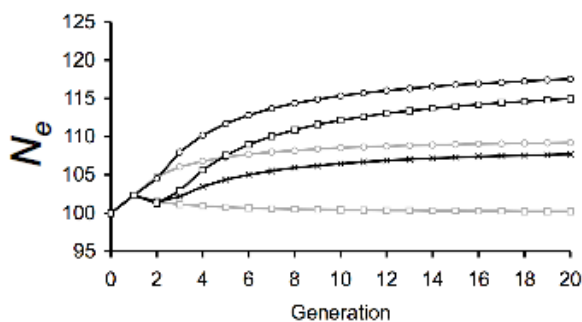

(d)

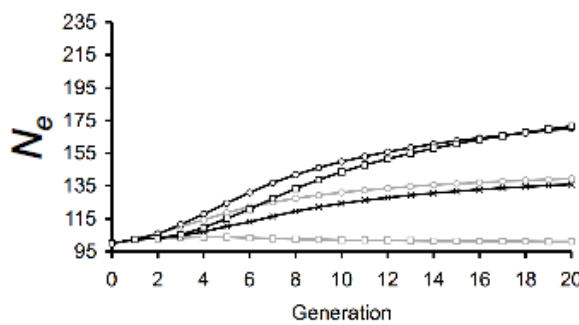


Figure 4. The effect of introducing individuals containing Trojan sex chromosomes $(N=5)$ into a small wild population $\left(\mathrm{N}_{\mathrm{MXY}}=50, \mathrm{~N}_{\mathrm{FXX}}=50\right)$ on $N_{a}(\boldsymbol{a}, \boldsymbol{c})$ and $N_{e}(\boldsymbol{b}, \boldsymbol{d})$ and. Trojan individuals $\left(\mathrm{N}_{\mathrm{M} \mathrm{XX}}\right)$ were introduced during $(a, b)$ a single episode in generation $t=1$, and $(c, d)$ for five generations $(t=1$ to 5). Female productivity was set at $n=2$ offspring. Changes in population size are depicted after introduction of XX males (squares, black line). For comparison, growth curves resulting from XX female (circles, grey line) and $\mathrm{XY}$ male (squares, grey lines) introduction (extra $\mathrm{N}_{\mathrm{FXX}}$ and $\mathrm{N}_{\mathrm{MXY}}=5$ ) are also depicted.

(a)

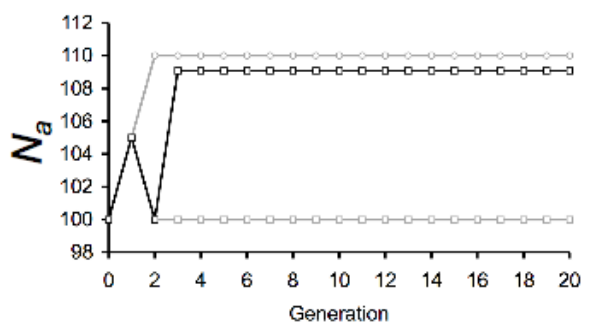

(c)

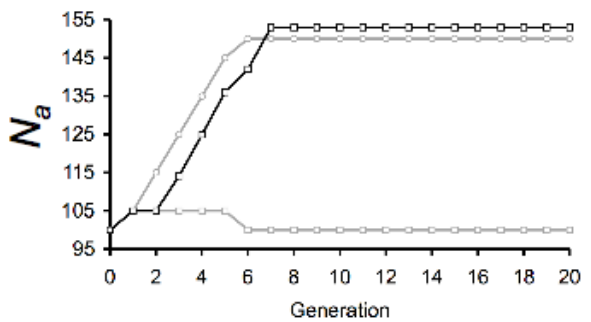

(b)

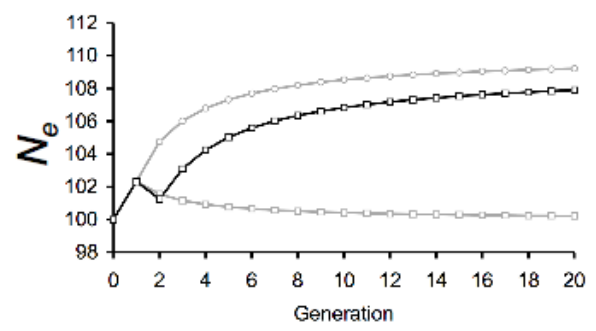

(d)

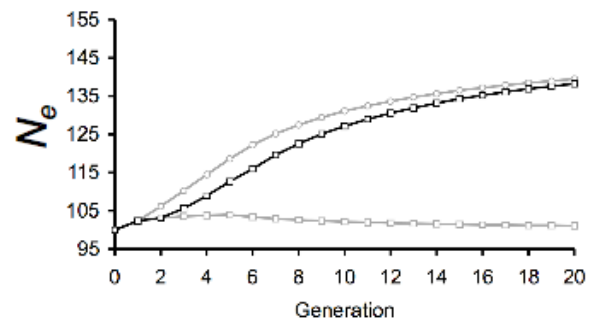


Figure 5. The influence of the number of introduced individuals on $N_{e}$ after 20 generations following $(\boldsymbol{a})$ a single release episode during generation $t=1$, and (b) multiple releases over five generations ( $t=1$ to 5 ). Bars represent $\mathrm{ZZ}$ males (white, hatched), ZW females (white), ZW males (mid grey, hatched), WW males (dark grey, hatched) and WW females (dark grey).

(a)

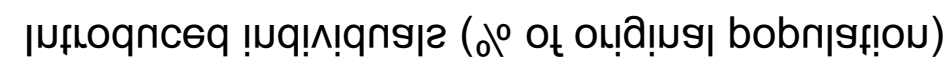

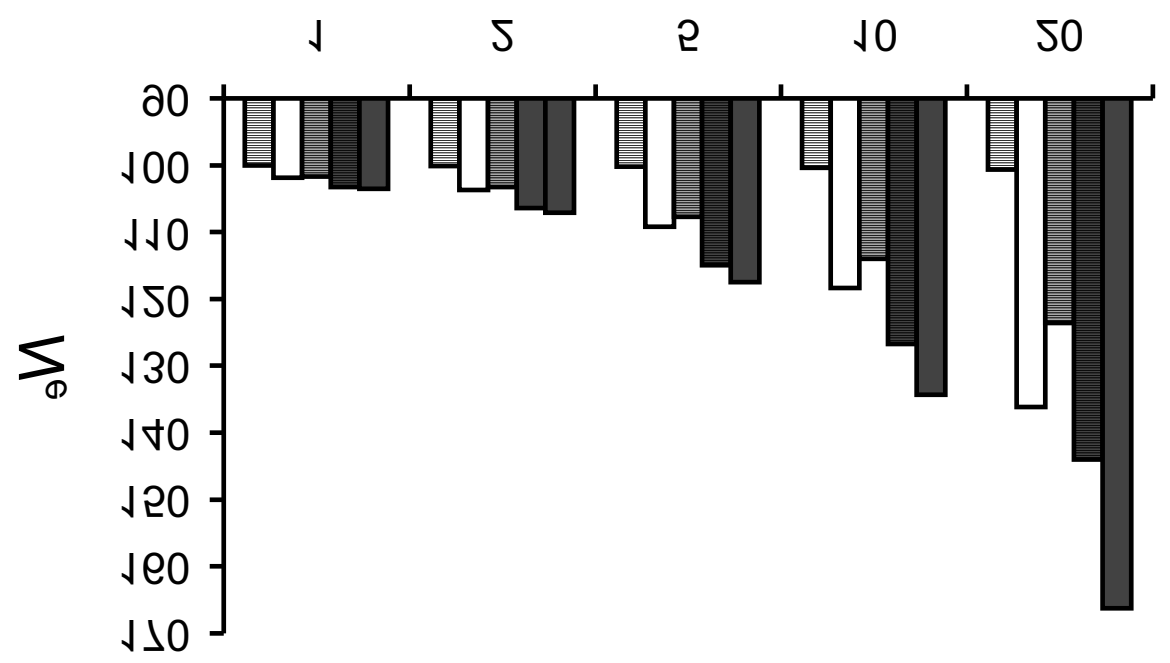

(b)

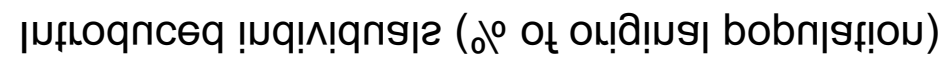

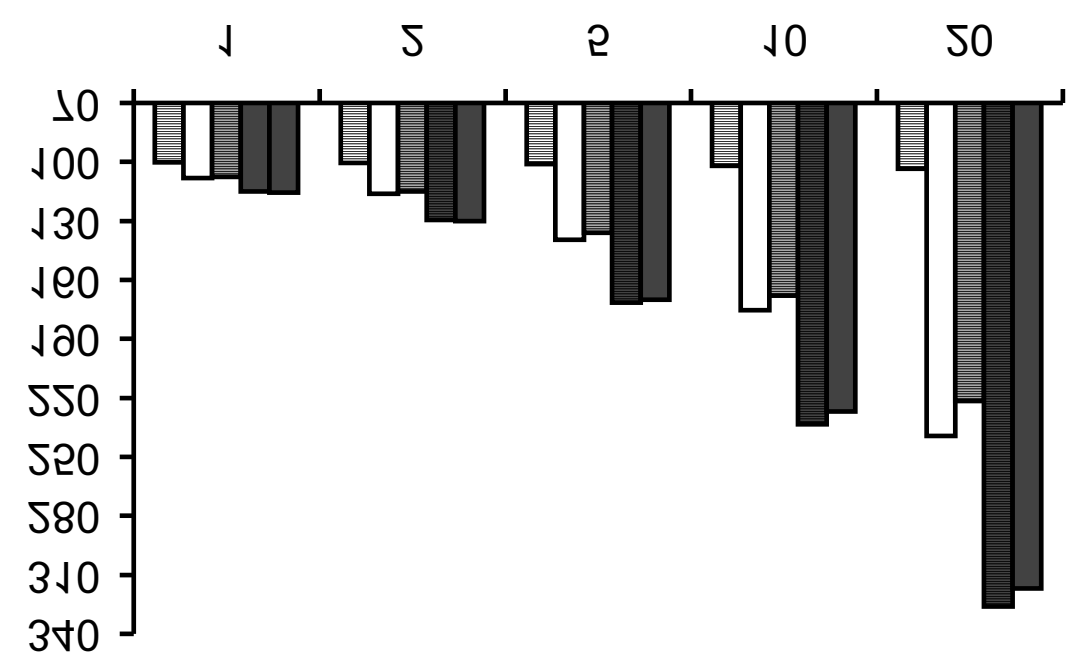


Figure 6. The effect of introducing individuals containing Trojan sex chromosomes on the sex ratio of the wild population. Trojan individuals were introduced during $(\boldsymbol{a})$ a single episode in generation $t=1$, and $(\boldsymbol{b})$ for five generations ( $t=1$ to 5 ). Sex ratios are depicted for introduction of $0.05 N_{a} Z W$ males (crosses, black line), WW females (circles, black line) and WW males (squares, black line). For comparison, sex ratios resulting from ZW female (circles, grey line) and ZZ male (squares, grey lines) introduction are also depicted.

(a)

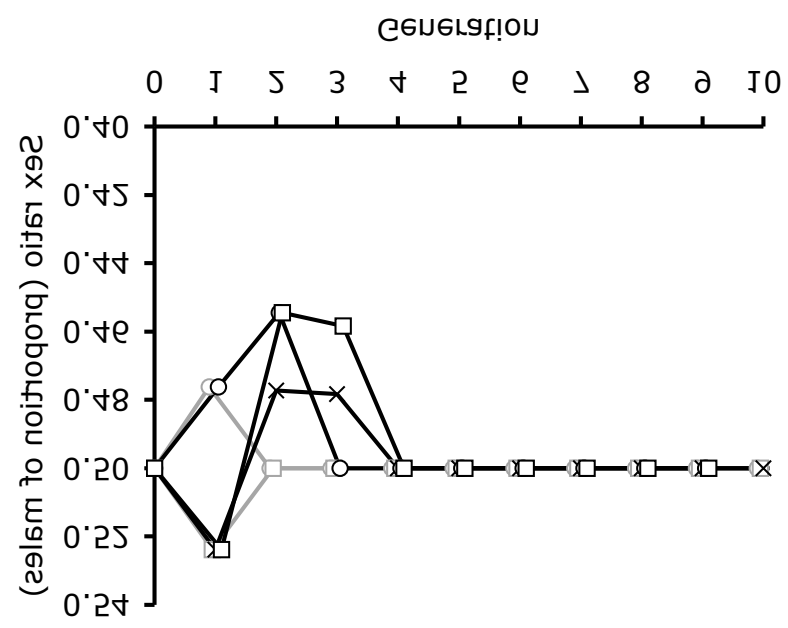

(b) 
Figure 7. The effect of changes in relative viability on the success of single $(\boldsymbol{a}$ $c)$ and multiple $(d-f)$ introductions of $0.05 N_{a}$ after generation $t=20 . N_{e} \mathrm{ZW}$ males $(\boldsymbol{a}, \boldsymbol{d}), \mathrm{WW}$ females $(\boldsymbol{b}, \boldsymbol{e}), \mathrm{WW}$ males $(\boldsymbol{c}, \boldsymbol{f})$. ZW intro $=$ dotted line. Lines (from black to thin grey) represent the effect on $N_{e}$ of $p$ for values of 1.0, 0.8, $0.6,0.4,0.2$ and 0 , respectively.

(a)

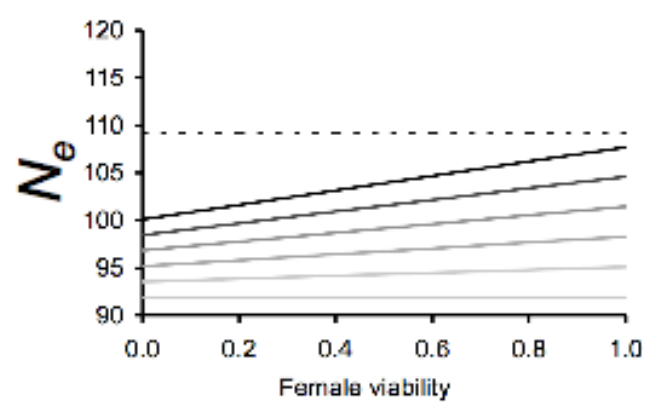

(b)

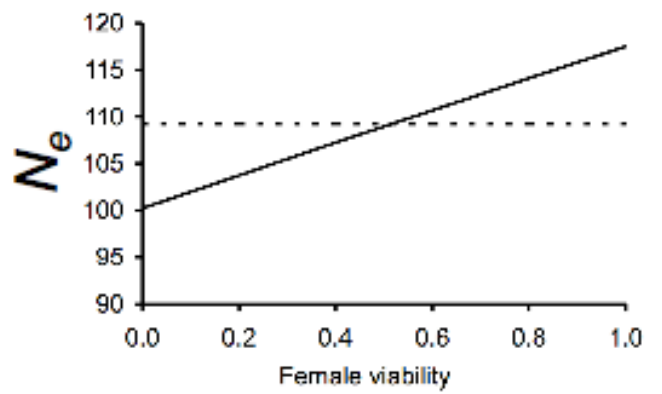

(c)

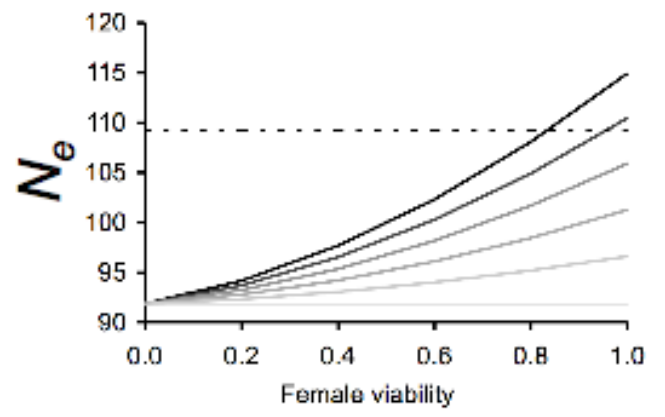

(d)

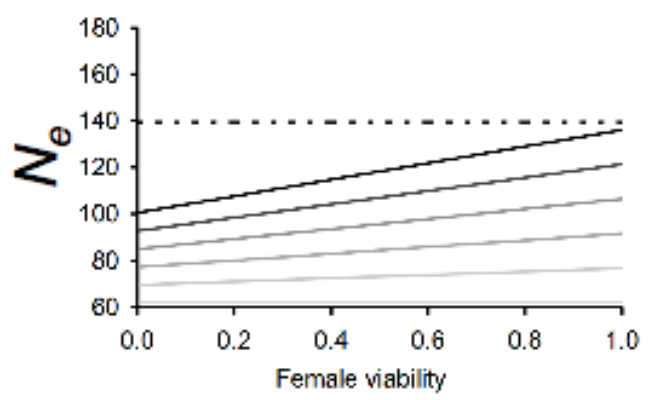

(e)

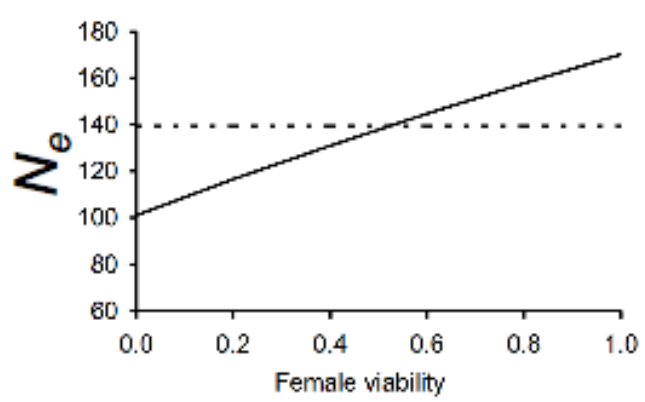

$(f)$

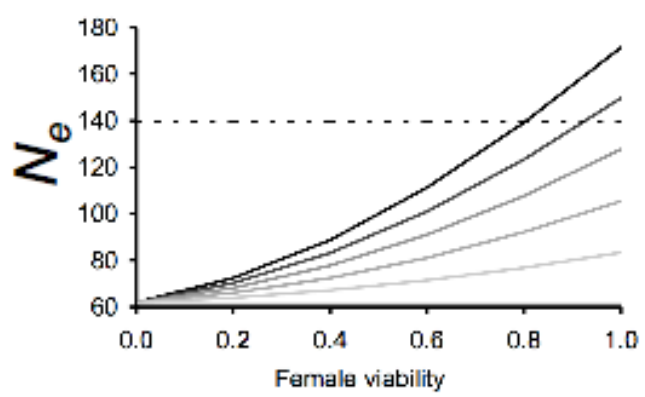

